\title{
An Improved RF Detection Algorithm Using EMD-based WT
}

Xue Lv* and Zekun Wang

${ }^{1}$ Wuhan Huaxia University of Technology, No 589 Guanshan Avenue, Hongshan, Wuhan 430223, China [e-mail: lvxueer200@163.com; 46595435@qq.com] *Corresponding author: Xue Lv

Received November 6, 2018; revised December 30, 2018; accepted January 29, 2019; published August 31, 2019

\begin{abstract}
More and more problems for public security have occurred due to the limited solutions for drone detection especially for micro-drone in long range conditions. This paper aims at dealing with drones detection using a radar system. The radio frequency (RF) signals emitted by a controller can be acquired using the radar, which are usually too weak to extract. To detect the drone successfully, the static clutters and linear trend terms are suppressed based on the background estimation algorithm and linear trend suppression. The principal component analysis technique is used to classify the noises and effective RF signals. The automatic gain control technique is used to enhance the signal to noise ratios (SNR) of RF signals. Meanwhile, the empirical mode decomposition (EMD) based wavelet transform (WT) is developed to decrease the influences of the Gaussian white noises. Then, both the azimuth information between the drone and radar and the bandwidth of the RF signals are acquired based on the statistical analysis algorithm developed in this paper. Meanwhile, the proposed accumulation algorithm can also provide the bandwidth estimation, which can be used to make a decision accurately whether there are drones or not in the detection environments based on the probability theory. The detection performance is validated with several experiments conducted outdoors with strong interferences.
\end{abstract}

Keywords: principal component analysis, automatic gain control, empirical mode decomposition, wavelet transform

This work was funded by the Nature Science Foundation of China (41527901, 61501424 and 61701462). 


\section{Introduction}

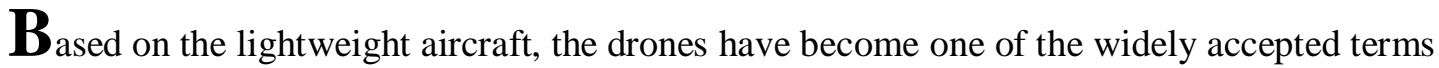
integrated with different devices, which are operated without a human pilot onboard in conjunction with ground control systems [1]. As a useful tool, the drones have are used in military applications initially including search/destroy missions [2], surveillance, aerial photography, and reconnaissance [3], which can provide higher spatial resolution such as up to the sub-centimeter level in small areas due to their low operational altitude [4]. The relatively low cost of different drone platforms is the most important factor, which leads to their wide popularity and recent success. Over the previous decade, drones have been increasing, which are used for data acquisition by more and more actors and stakeholders such as scientific institutions, governmental authorities, individuals and commercial operators [5]. Especially in civil applications, drones are widely applied in land administration [6], documentation of cultural heritage and archaeological sites [7], support for disaster management [8], high-resolution surface reconstruction in the geosciences [9], forest and agriculture change detection [10], surveying and mapping [11], and wildlife observation [12].

Drones can provide more and more convenient services, but numerous problems for the public security come correspondingly. As known, one drone was used to interrupt the US Open tennis match and another crashed at the White House. The drones in illegal flight disturb the air flight conditions and pose a great threat to the flight safety of passenger aircraft. Some small drones are usually used for invading human privacies. More and more accidents have been trigged due to the popularity of drones. Until now, shooting the drones failing to comply with regulatory requirements is considered as the most effective solution. The authors in [13-14] employ the genetic algorithm (GA) to jam the drones. In [15], the GPS signals are jamming, which can make the drone disable. However, all these methods can only work better only when these drones are detected, which are impossible to achieve without effective detection solution. Thus, more works are required to deal with the public security. Many detection solutions have been developed in past few years including the acoustic recognition, active radar, visual recognition, infrared spectrum analysis, and RF detection [16-20]. By modulating the RF signals, the active radar was used for drone detection at short ranges [16]. Without considering the flight pattern, the drones are recognized using a calibrated radar cross section technique in [17] at $2000 \mathrm{~m}$. The authors employed the complex-log Fourier transform method for drone detection, which can acquire the spectrogram features [18]. Based on the log-polar transformations and space-variant resolution, the drones are identified [19], which can only work at short range. The drones are located using the acoustic array in [20] at an effective range of $300 \mathrm{~m}$. Meanwhile, the detection algorithms based on neural networks (NN) [21], fuzzy systems [22], least squares (LS) [23], frequency based approaches [24], Kalman filters [25], and Gauss-Newton [26] etc. have also been developed. However, limited works can be acquired for drone detection. Thus, some alternative methods have to be developed to deal with the problems of drones in illegal flight. This paper mainly deals with the weak signal extraction solution for the drone detection. The major factors including the static clutter and linear trend term are removed. The principal component analysis is used to acquire the effective radio frequency (RF) signals. And the signal to noise ratios (SNR) of RF signals is improved based on the automatic gain control algorithm. The empirical mode decomposition (EMD) based wavelet transform (WT) is employed to reconstruct the effective RF signals. Based on the statistic theory, the azimuth information between the radar and drone is acquired. 
The bandwidth of RF signals is obtained using the developed accumulation algorithm. Using the probability theory, accurate judgements can be made whether there are drones or not in the detection environments.

The following sections are introduced as: the system model is introduced in Sec. 2. Sec. 3 discusses the proposed detection algorithm using passive radar. The detection results are discussed in Sec. 4. Sec. 5 concludes the whole paper.

\section{System Model}

As shown in Fig. 1, a drone, a receiver, and a controller are considered in the developed passive radar system. The controller can emit RF signals, which can meet the requirements of communicating with the drone. The receiver can acquire RF signals emitted by the controller. As known, RF signals are usually within $2.4-2.4835 \mathrm{GHz}$, which can control the most drones for flight and image transmission based on the frequency hop spread spectrum technique [27]. Meanwhile, the RF signals have to meet the IEEE 802.11 standard [28].

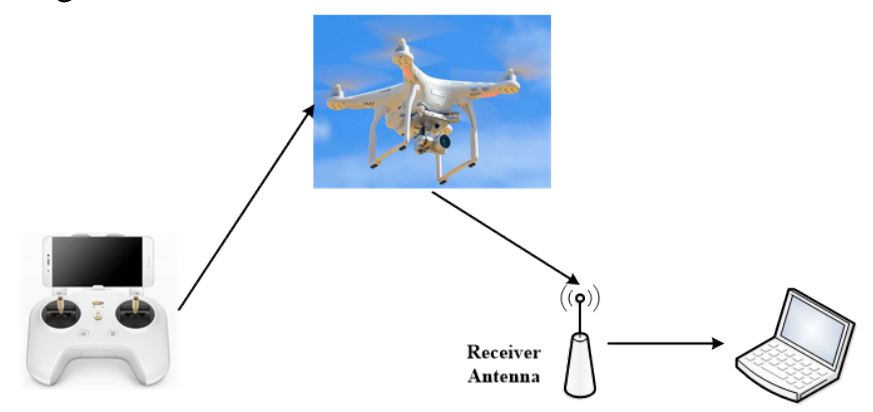

Fig. 1. The system model.

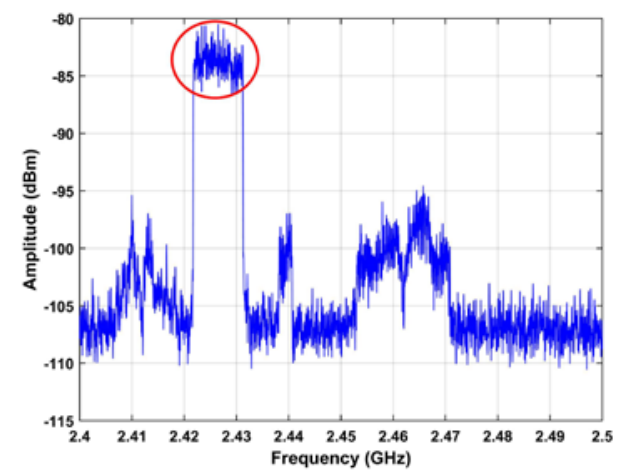

Fig. 2. The spectrums of RF signals.

The used drone and the corresponding controller are developed by the DJ-Innovations. Using the receiver, the data can be acquired for each given distance such as $2500 \mathrm{~m}$. Assuming that the power of the received RF signals is given by $\mathrm{y}(f)$ in $\mathrm{dBm}$, where $f$ denotes the frequency samples within 2.4-2.5 GHz. All 5120 samples can be acquired with the interval of $f_{\mathrm{w}}=19.53 \mathrm{kHz}$ as shown in Fig. 2. The results indicate that the RF signal emitted by the controller has a larger bandwidth, which is approximately $9.8 \mathrm{MHz}$. In real detection environment, due to the larger range between the receiver and the drone, the RF signals are usually too weak to find especially in complex multipath environments. This paper aims at the drone detection outdoors. 


\section{Proposed Algorithm}

Fig. 3 shows the proposed algorithm based on the acquired RF signals $\mathbf{A}_{M \times N}$ at $500 \mathrm{~m}$ using the radar, which is an azimuth-frequency matrix as show in Fig. 4.

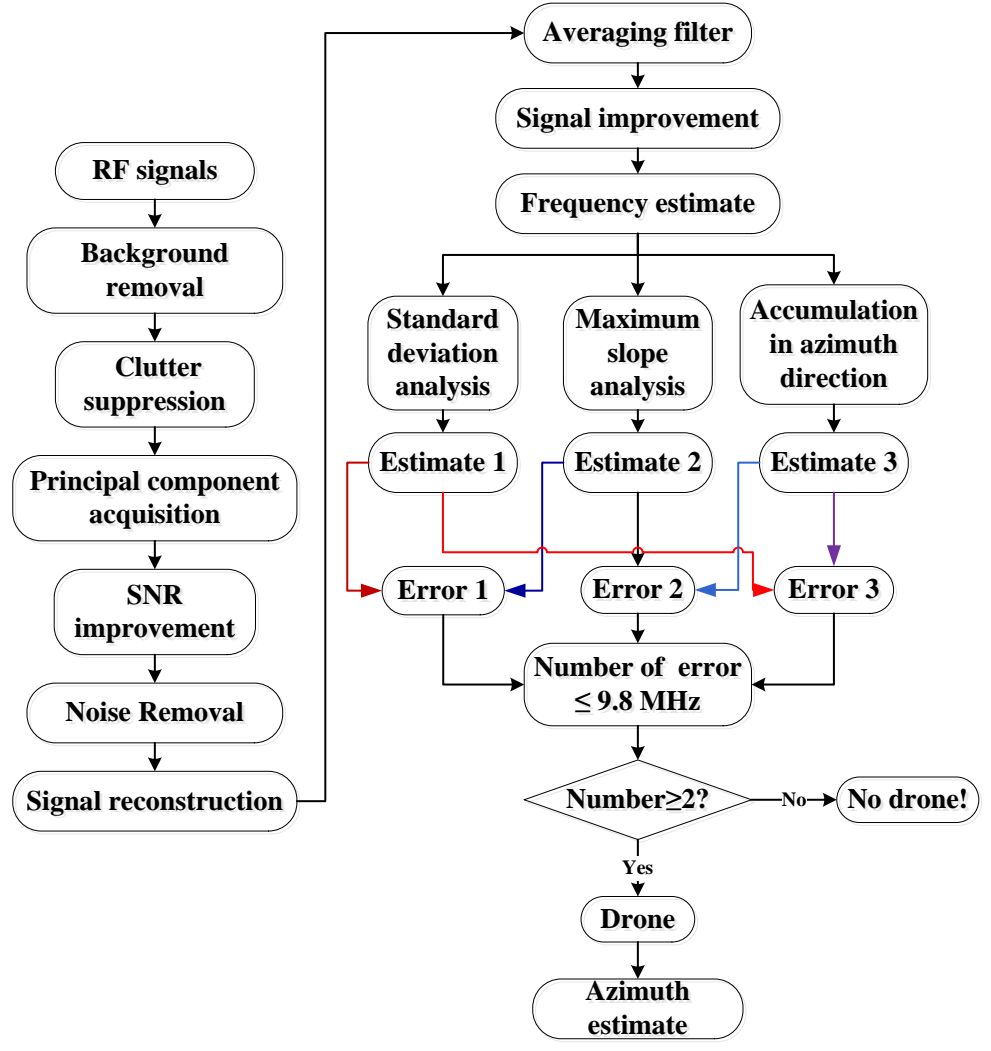

Fig. 3. The proposed detection algorithm.

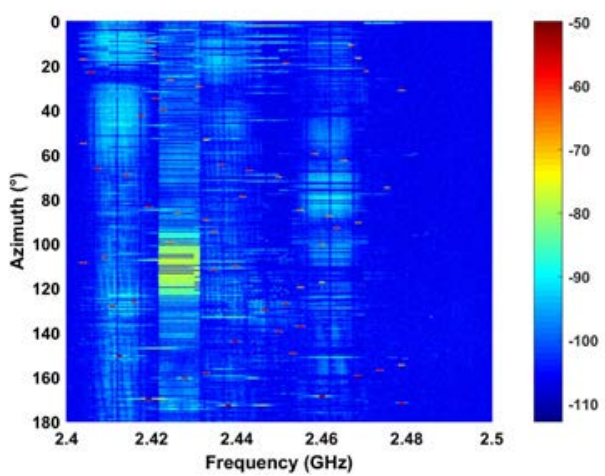

Fig. 4. The acquired RF signals at $500 \mathrm{~m}$.

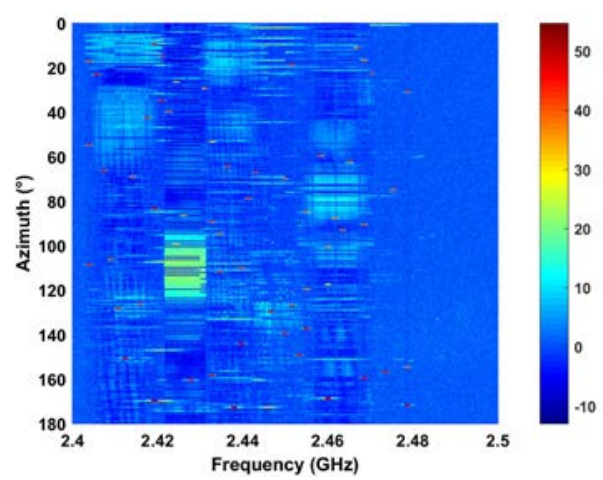

Fig. 5. The results removing static clutter.

\subsection{Static Clutter Removal}

The static clutter in $\mathbf{A}_{M \times N}$ [29] can be estimated as

$$
B=\frac{1}{M \times N} \sum_{m=1}^{M} \sum_{n=1}^{N} A[m, n]
$$

Subtracting (1) for each value in $\mathbf{A}$, the results $\mathbf{C}$ are acquired as shown in Fig. $\mathbf{5}$. 


\subsection{Linear Trend Suppression}

This subsection employs linear trend suppression to eliminate the potential terms with linear trend. As shown in Fig. 6, using the linear trend suppression, the results are [30]

$$
\mathbf{D}=\mathbf{C}^{\mathrm{T}}-\mathbf{X}\left(\mathbf{X}^{\mathrm{T}} \mathbf{X}\right)^{-1} \mathbf{X}^{\mathrm{T}} \mathbf{C}^{\mathrm{T}}
$$

where

$$
\mathbf{X}=\left[\begin{array}{ll}
\mathbf{x}_{1} & \mathbf{x}_{2}
\end{array}\right]=\left[\begin{array}{cc}
0 & 1 \\
2 & 1 \\
\vdots & \vdots \\
N-1 & 1
\end{array}\right]
$$
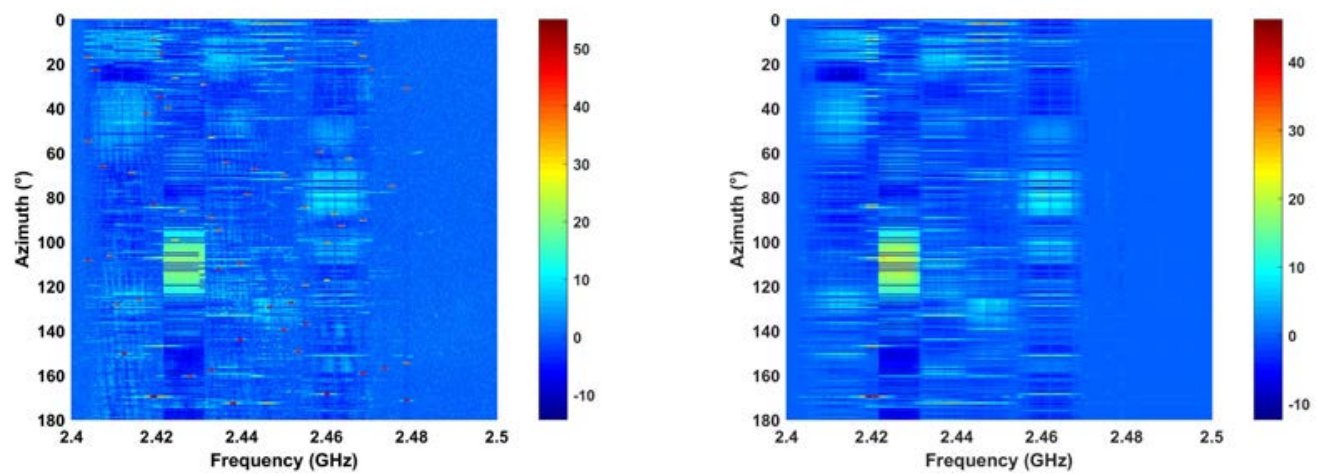

Fig. 6. The results using linear trend suppression. Fig. 7. The results using principal component analysis.

\subsection{Principal Component Analysis}

The principal component analysis is used to acquire RF signals. Usually, (2) can be divided into several complementary subspaces as [31]

$$
\mathbf{D}=\mathbf{U S V}^{\mathrm{T}}
$$

where $\mathbf{S}$ denotes a diagonal matrix, $\mathbf{U}_{M \times M}$ denotes an unitary matrix, and $\mathbf{V}_{N} \times_{N}$ is an unitary matrix. The singular values $\sigma_{i}$ in $\mathbf{S}$ meet $\sigma_{1} \geq \sigma_{2} \geq \sigma_{3} \geq \ldots \geq \sigma_{r} \geq 0$. The columns in $\mathbf{U}$, i.e. the eigenvectors of $\mathbf{W} \mathbf{W}^{\mathrm{T}}$, are composed of the left singular vectors. The columns in $\mathbf{V}$, i.e. the eigenvectors of $\mathbf{W}^{\mathrm{T}} \mathbf{W}$, are composed of the right singular vectors. Equation (4) can be redefined as

$$
\begin{aligned}
\mathbf{D} & =\sigma_{1}\left(\begin{array}{c}
\vdots \\
\mu_{1} \\
\vdots
\end{array}\right)\left(v_{1} \cdots\right)+\sigma_{k}\left(\begin{array}{c}
\vdots \\
\mu_{k} \\
\vdots
\end{array}\right)\left(\cdots v_{k} \cdots\right)+\cdots+\sigma_{N}\left(\begin{array}{c}
\vdots \\
\mu_{N} \\
\vdots
\end{array}\right)\left(\cdots v_{N} \cdots\right) \\
& =\mathbf{M}_{1}+\mathbf{M}_{2}+\cdots+\mathbf{M}_{k}+\cdots+\mathbf{M}_{G}
\end{aligned}
$$

where $\mathbf{M}_{\mathrm{k}}$ is the $k$ th intrinsic image with the same dimensions as $\mathbf{E}$.

As usual, (5) can be divided into two parts as

$$
\mathbf{D}=\mathbf{M}_{\text {drone }}+\mathbf{M}_{\text {Noise }}
$$


where $\mathbf{M}_{\text {drone }}$ is the RF signals, $\mathbf{M}_{\text {Noise }}$ is noises, which can be removed. Thus, the effective RF signals can be acquired as

$$
\mathbf{E}=\sum_{i=1}^{n} u_{i} \sigma_{i i} v_{i}^{\mathrm{T}}
$$

Fig. 7 shows the results based on the principal component analysis algorithm.

\subsection{Automatic Gain Control}

As an adaptive algorithm, the automatic gain control algorithm is extensively used in many applications [32]. The gain values of the inputs in a given range usually can be adjusted to appropriate levels using the fed levels of the averaged outputs.

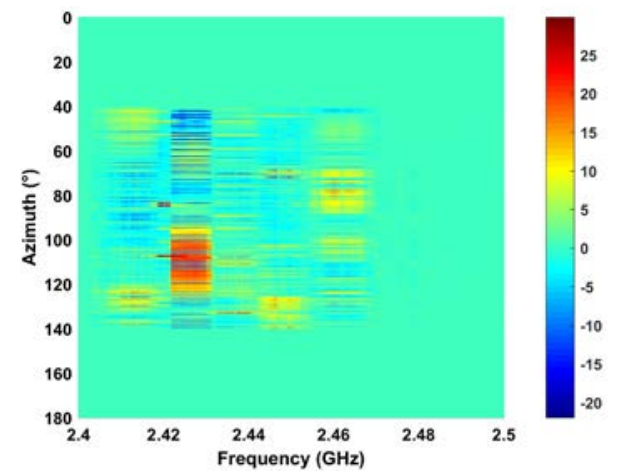

. Fig. 8. The results using automatic gain control.

An effective application of automatic gain control method is to enhance the amplitudes of the weak signals in radar systems [33]. Via adjusting the gain values based on the calculated power of the signals in a window $w$, the key is to compare the gain values and $g_{\max }$ as

$$
g_{\text {mask }}= \begin{cases}g_{\max } & g_{\text {norm }}[i, n] \geq g_{\max } \\ g_{\text {norm }}[i, n] & g_{\text {norm }}[i, n]<g_{\max }\end{cases}
$$

where $n=0,2, \cdots, N-1, i=0,1, \cdots, M-w$.

The normalized gain values $g_{\text {norm }}[i, n]$ are

$$
g_{\text {norm }}[i, n]=g[i, n] / g_{\min }[i, n]
$$

where $g_{\min }[i, n]$ is the acquired minimal gain, which can be expressed as

$$
g[i, n]=w / e[i, n]
$$

where $e[i, n]$ is the calculated power of the signals in a window $w$, which can be expressed as

$$
e[i, n]=\sqrt{\sum_{k=i}^{w+i-1} C[k, n]^{2}}
$$


Using the automatic gain control method, $g_{\max }$ can be determined based on the calculated gain values. In this paper, $g_{\text {norm }}[i, n]$ is set to be 0.6 based on the empirical evidences [34].

We can acquire the following results given by

$$
F[i, n]=E[i, n] \times g_{\text {mask }}[i, n]
$$

The results using the automatic gain control algorithm are shown in Fig. 8. We can see RF signals are improved significantly, which can make it easy to detect.

\subsection{Noise Suppression}

As an adaptive algorithm, EMD is widely used to deal with the non-static signals [35], which can make it divided into various intrinsic mode functions (IMFs) and a residual trend.
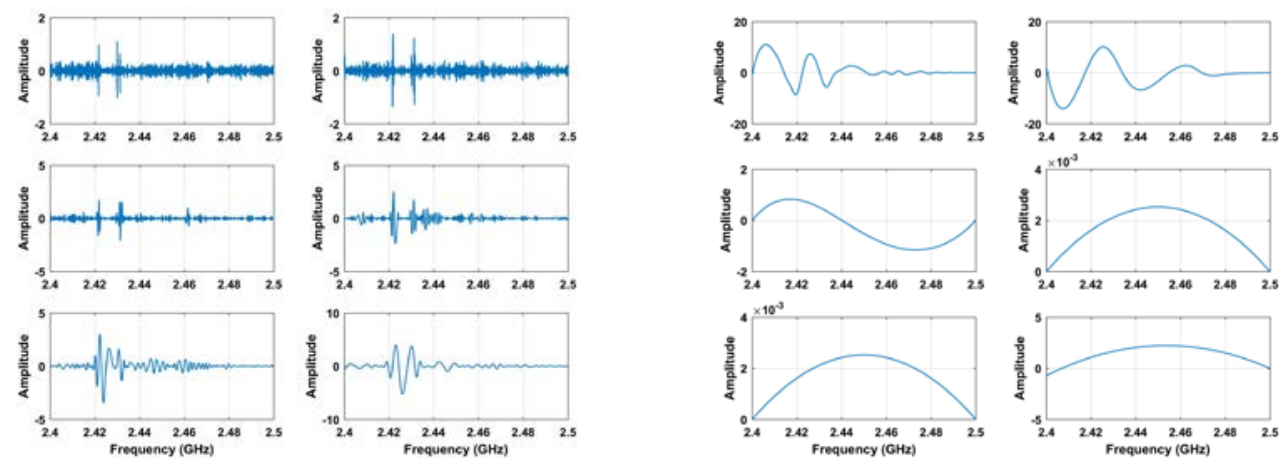

(a)
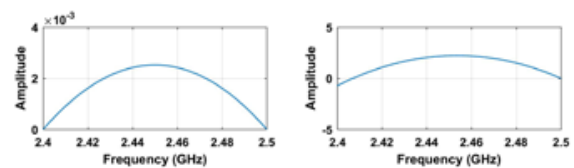

(b)

Fig. 9. The acquired IMFs using EMD algorithm.

Fig. 9 shows the first 12 IMFs of the RF signals and a residual trend. The residual trend is considered as noises. For the IMFs, the boundary between RF signals and noises is

$$
R P_{v}=\left|\frac{P_{v}-\frac{1}{v-1} \sum_{i=1}^{v-1} P_{i}}{\frac{1}{v-1} \sum_{i=1}^{v-1} P_{i}}\right|, \quad v \geq 2
$$

where

$$
\begin{gathered}
P_{v}=E_{v} \bar{T}_{v} \\
E_{v}=\frac{1}{N_{v}} \sum_{j=1}^{N_{e}}\left|C_{v}(j)\right|^{2} \\
\bar{T}_{v}=2 N_{v} / O_{v}
\end{gathered}
$$

and $N_{v}$ is the number of values in $I M F_{v}, C_{v}$ is the maximum value in $I M F_{v}$, and $O_{v}$ is the number of local extrema in $I M F_{v}$. For AWGN, (14) is approximately a constant. As a result, $I M F_{v}$ can be regarded as noises when (13) < 1 . It was determined by simulation that IMFs 1 to 6 can be considered as noises. Using EMD, the results are shown in Fig. 10, which indicate the RF signals are improved obviously. 


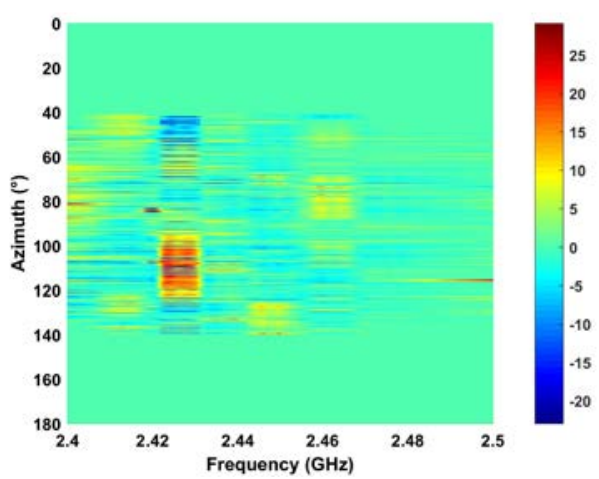

Fig. 10. The results using EMD

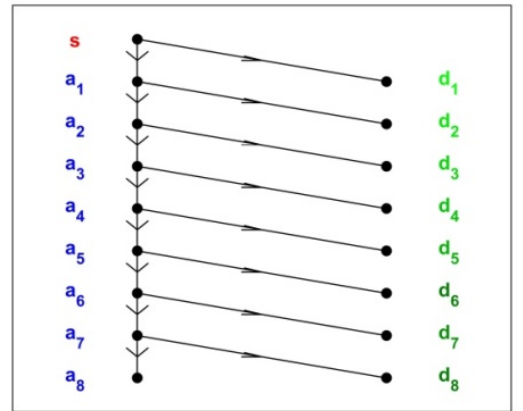

Fig. 11. Eight levels wavelet packet decomposition tree.

\subsection{Signal Reconstruction}

This subsection employs WT to reduce influences of noises and reconstruct RF signals [36-37]. In wavelet analysis, RF signals for each given azimuth $G_{m}(n)$, are used to compare with the wavelet function. The acquired coefficients can be used for showing how RF signals and the wavelet functions match. Due to RF signals are stored in digital form, the coefficients obtained from the discrete WT are

$$
\mathrm{T}=\frac{1}{\sqrt{a}} \sum_{n} G_{m}(n) \psi *\left(\frac{k-b}{a}\right)
$$

where $k$ denotes the samples of RF signals, * denotes the complex conjugate, $a$ is the scaling parameters, $b$ is the translation parameters of the mother wavelet $\Psi(n)$, and $\psi((k-b) / a)$ is the son wavelets.
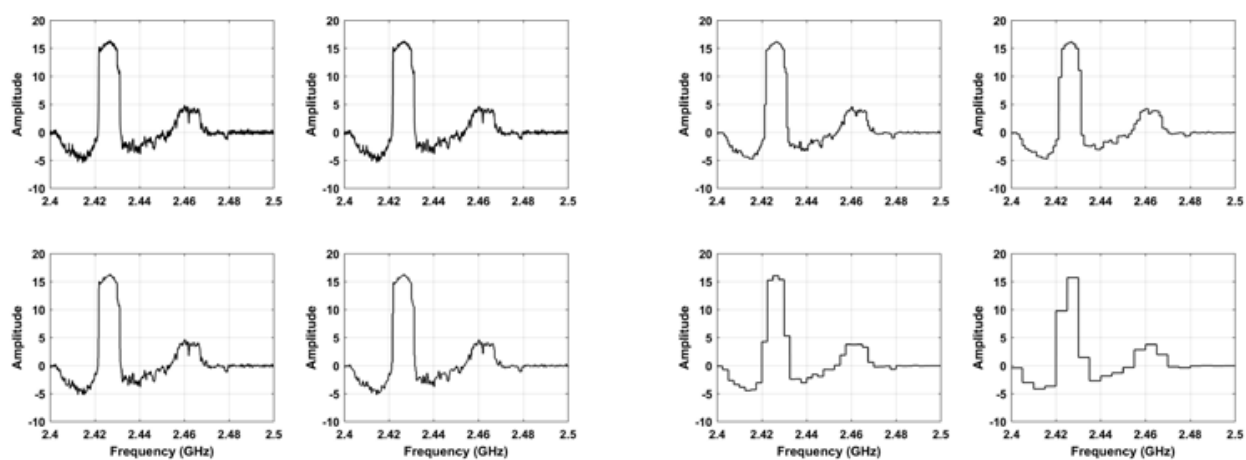

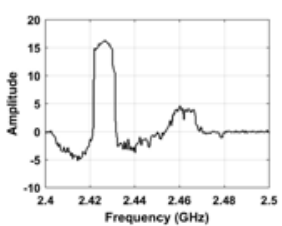

(a)
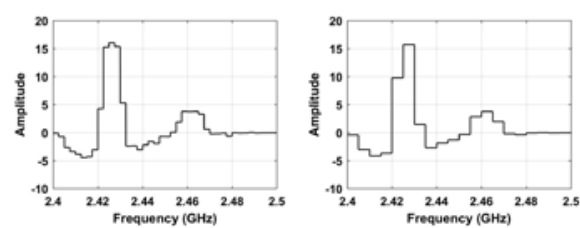

(b)

Fig. 12. The decomposed signals using WT technique.

In this paper, the Haar wavelet is considered as mother wavelet due to its simple numerical implementation and simplified computation. By employing a wavelet packet decomposition tree with eight levels as shown in Fig. 11, the decomposed RF signals are shown in Fig. 12. Based on the spectrums of Welch power of RF signals, they can be reconstructed from the decomposed signals $d_{4}$ as shown in Fig. 12. Fig. 13 shows the reconstructed signals $\mathrm{H}$. 


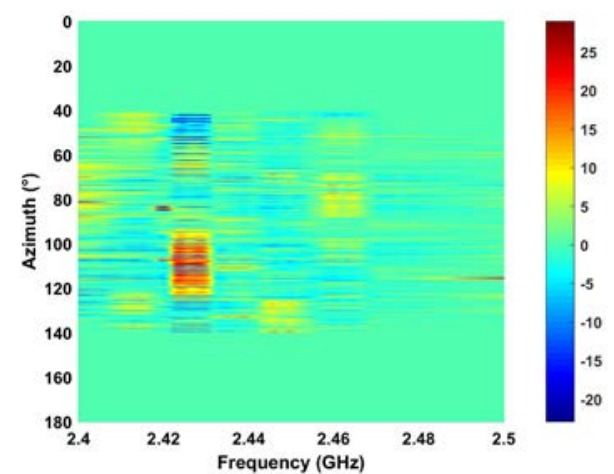

Fig. 13. The results using WT.

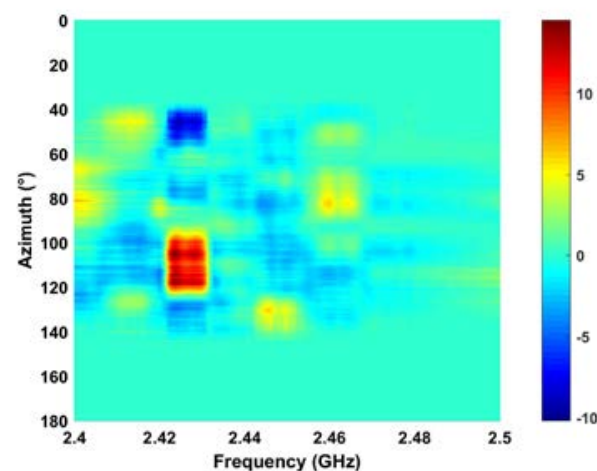

Fig. 14. The results using averaging filter.

\subsection{Averaging Filter}

This subsection proposes an improved filter to improve SNR. The following results can be obtained when the filter is performed on $\mathbf{H}$ in azimuth direction as

$$
I_{m}(k)=\frac{1}{12} \sum_{n=12 k}^{13 k-1} H_{m}(n)
$$

where $k=1, \cdots,\lfloor M / 12\rfloor .\lfloor M / 12\rfloor$ denotes the maximum integer value less than $M / 12$.

Fig. 14 shows the results based on the filter, which show RF signals are improved effectively and the better performance on noises suppression.

\subsection{Frequency Estimation}

This subsection proposes three methods for frequency estimations of RF signals.

Firstly, the Hilbert transform is used to improve SNR. For each azimuth signal $I_{\mathrm{m}}(n)$, the Hilbert transform is applied [37], which is given by

$$
J_{m}(n)= \begin{cases}2 I_{m}(n) & \omega(n)>0 \\ I_{m}(n) & \omega(n)=0 \\ 0 & \omega(n)<0\end{cases}
$$

where $\omega$ denotes the frequency samples.

To acquire the frequency estimations, the characteristics including the skewness [38], kurtosis, standard deviation, and maximum slope are analyzed. For each azimuthal signal $J_{\mathrm{m}}(n)$, the standard deviation is given by

$$
K(m)=\sqrt{\frac{\sum_{n=1}^{N}\left(J_{m}(n)-\mu_{m}\right)^{2}}{N-1}} \quad m=1,2, \cdots, M
$$

where $\mu_{m}$ denotes the mean value of $J_{m}(n)$.

And the maximum slope is expressed as

$$
L(m)=\max _{1 \leq i \leq N-m+1}\left\{\operatorname{linefit}\left(J_{m}(i), J_{m}(i+1), J_{m}(i+2), \cdots, J_{m}(i+n-1)\right)\right\}
$$


Using RF signals at $500 \mathrm{~m}$, the analyzed results are shown in Fig. 15. Thus, the frequency value corresponding to the maximum (20) is considered as the estimation $\tau_{1}$, which is

$$
\tau_{1}=\omega(\gamma)
$$

where

$$
\gamma=\underset{1 \leq i \leq M}{\arg \max }[K(m)]
$$

And the frequency value corresponding to maximum (21) is considered as the estimation $\tau_{2}$, which is

$$
\tau_{2}=\omega(\eta)
$$

where

$$
\eta=\underset{1 \leq i \leq M}{\arg \max }[L(m)]
$$

$\omega$ denotes the frequency samples.

Further, the accumulation method in azimuth direction is proposed to estimate the frequency, which is given by

$$
Q_{m}=\sum_{i=1}^{m} J_{n}(i) \quad n=1,2, \cdots, N
$$
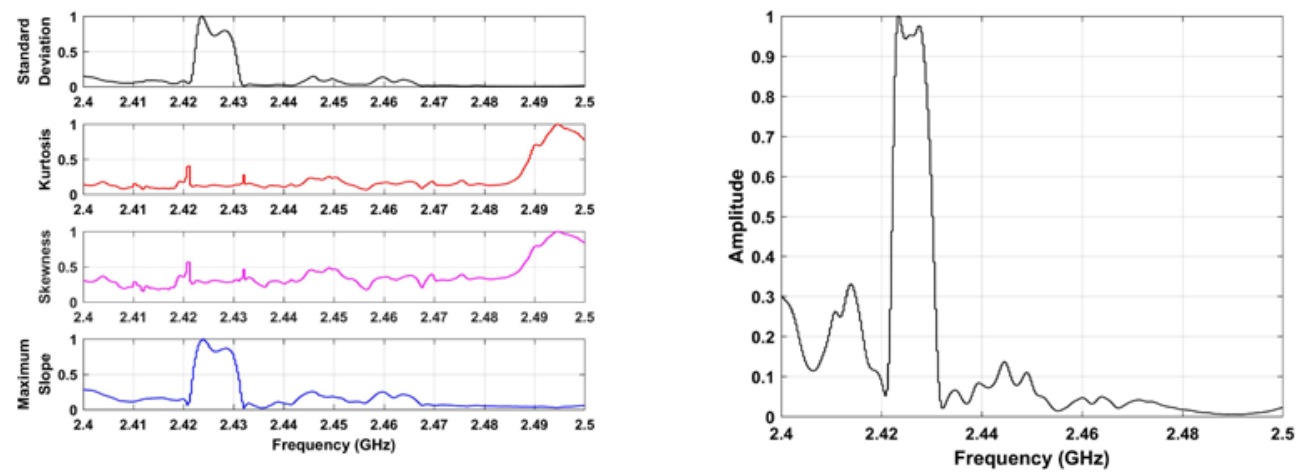

Fig. 15. The analyzed results of the RF signals.

Fig. 16. The results using the accumulation method.

As shown in Fig. 16, the maximum (22) is referred as the frequency estimation $\tau_{3}$, which is given by

$$
\tau_{3}=\omega(v)
$$

where

$$
v=\underset{1 \leq i \leq M}{\arg \max }\left[Q_{m}\right]
$$




\subsection{Target Determination}

To determine whether there are drones in the detection environment or not, the errors calculated from the three frequency estimates are employed as

$$
\alpha_{i j}=\left|\tau_{i}-\tau_{j}\right| \quad\left\{\begin{array}{l}
i=1,2,3 \\
j=1,2,3 \\
i \neq j
\end{array}\right.
$$

Due to the bandwidth of RF signals is about $10 \mathrm{MHz}$, the calculated errors can be used as a threshold to determine whether there are drones in the detection environments. Based on the three frequency estimates, three errors can be acquired. There are drones when $\lambda \geq 2$, while there are not drones when $\lambda<2$. $\lambda$ denotes the number of the error which is larger than $10 \mathrm{MHz}$.

\subsection{Azimuth Estimation}

To acquire the azimuth estimation, the standard deviation of each frequency signal is analyzed. For each frequency signal $J_{m}(n)$, the standard deviation is

$$
P(n)=\sqrt{\frac{\sum_{m=1}^{M}\left(J_{n}(m)-\mu_{n}\right)^{2}}{M-1}} \quad n=1,2, \cdots, N
$$

where $\mu_{n}$ denotes the mean value of $J_{m}(n)$.

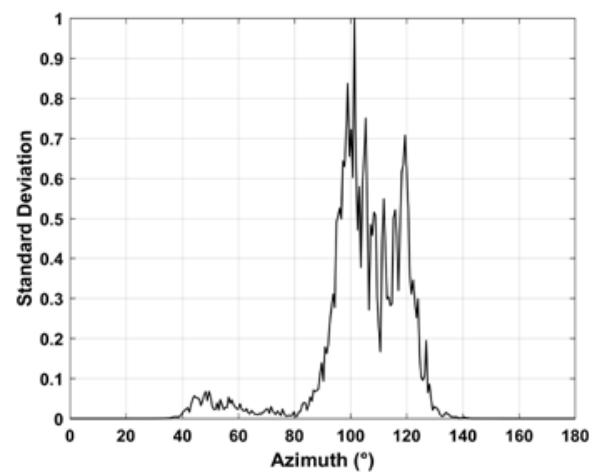

Fig. 17. The analyzed results of RF signals.

Using RF signals obtained at $500 \mathrm{~m}$, the analyzed results are shown in Fig. 17, which indicate the area with the normalized values larger than 0.4 are considered as the azimuth range.

\section{Results and Discussion}

Experimental results are compared with the minimum mean distance (MMD) classifier. Using the passive radar system, several experiments are conducted to validate the developed algorithm. (1) In the conducted first experiment, the used drone in the air is $100 \mathrm{~m}$ in height. The distance from the radar to drone is $500 \mathrm{~m}, 1500 \mathrm{~m}, 2400 \mathrm{~m}, 2500 \mathrm{~m}$, and $2800 \mathrm{~m}$ at horizontal direction. The conducted experiments are used to validate the better detection 
capability in long ranges. (2) The second experiment is carried out with the drone at a distance of $2400 \mathrm{~m}$ away from the radar. There are several interference sources in the detection environments. The conducted experiment is used for testing the performance of noise suppression using the developed algorithm. (3) In the third experiment, two drones are used as targets at different distances. The capability of multiple drone detection is validated.

\subsection{Detection Performance at Different Distances}

This subsection validates the performances of the developed detection algorithm using RF signals obtained at different distances including $500 \mathrm{~m}, 1500 \mathrm{~m}$, and $2400 \mathrm{~m}$, which are shown in Figure 18. We can see RF signals can only be extracted obviously in short range. However, RF signals are too weak to identify in long range such as $2400 \mathrm{~m}$ especially with strong interferences.

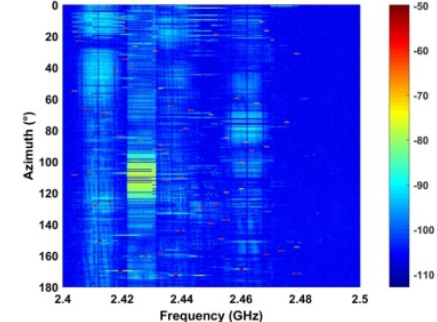

(a)

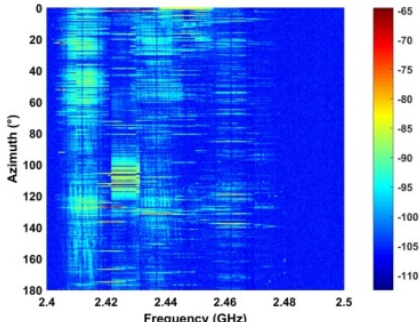

(b)

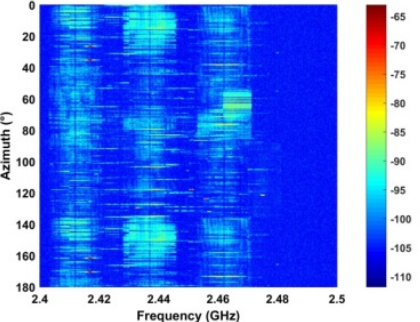

(c)

Fig. 18. The acquired RF signals at (a) $500 \mathrm{~m}$; (b) $1500 \mathrm{~m}$; and (c) $2400 \mathrm{~m}$.

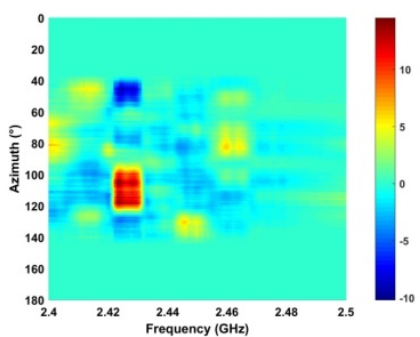

(a)

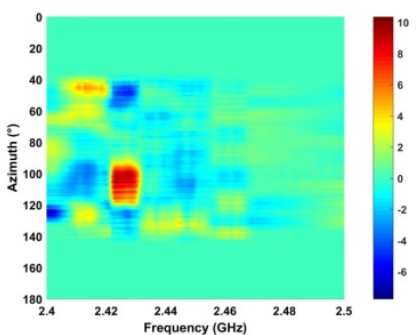

(b)

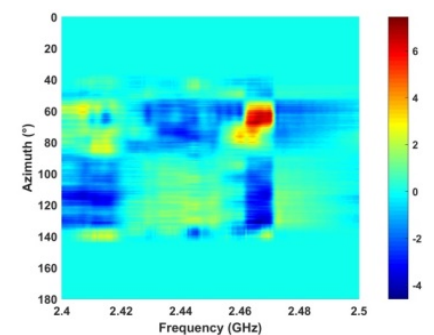

(c)

Fig. 19. The results after removing noises at (a) $500 \mathrm{~m}$; (b) $1500 \mathrm{~m}$; and (c) $2400 \mathrm{~m}$.

Based on the developed detection algorithm, the results after removing clutters are shown in Fig. 19, which indicate RF signals are improved significantly compared with the results in Fig. 18.

\subsection{Detection Performance Under Strong Interference}

The detection capability of the developed algorithm under strong interference environments is validated based on RF signals acquired at different distances including $2500 \mathrm{~m}$ and $2800 \mathrm{~m}$. In the detection environment, there are several interference sources as shown in Fig. 20. There are several interference sources i.e. the signals in the elliptical region with larger amplitudes than RF signals, which make drone more challenging to identify. The results after removing noises based on the developed algorithm are shown in Fig. 21. Based on the acquired results, the major interferences are suppressed effectively and RF signals are improved. 


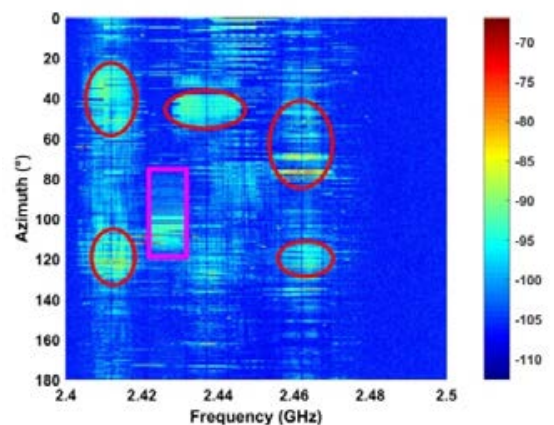

(a)

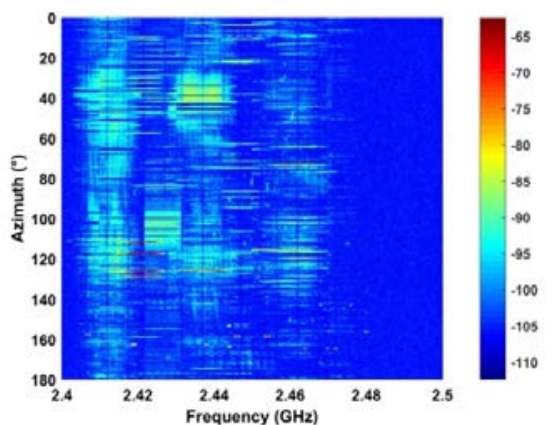

(b)

Fig. 20. The received RF signals at (a) $2500 \mathrm{~m}$; and (b) $2800 \mathrm{~m}$.

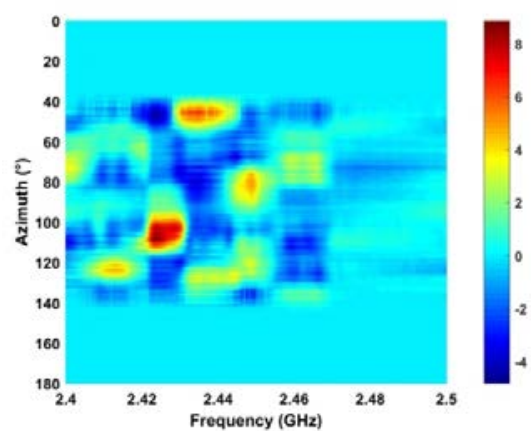

(a)

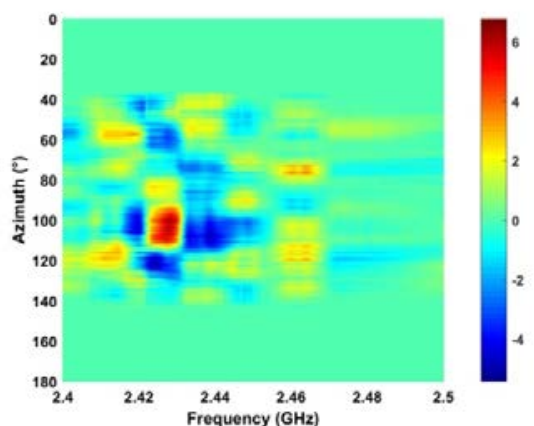

(b)

Fig. 21. The results after removing noises at (a) $2500 \mathrm{~m}$; and (b) $2800 \mathrm{~m}$

\subsection{Frequency Estimation}

The frequency estimates of RF signals at different distances based on the characteristics analysis algorithm are shown in Fig. 22.

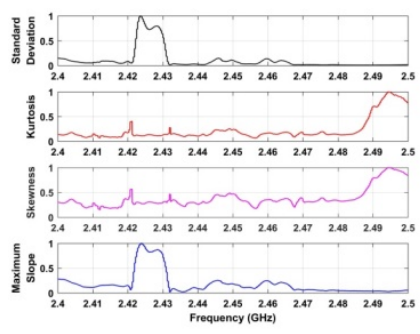

(a)

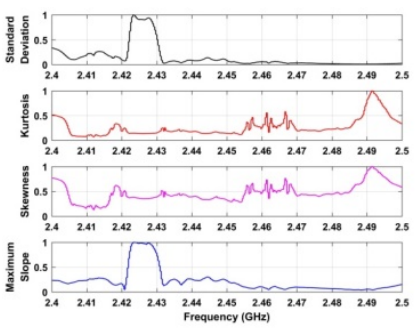

(b)

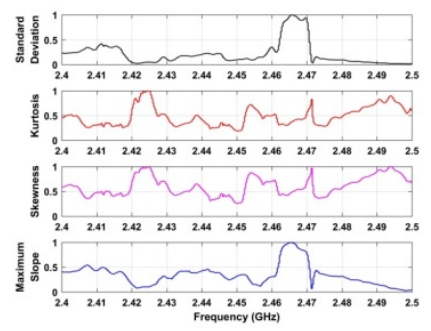

(c)

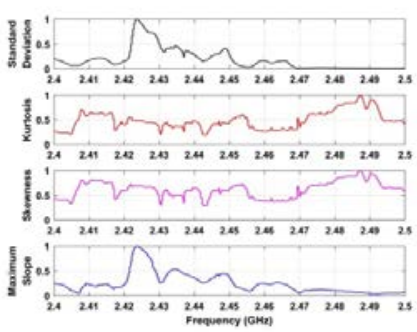

(d)

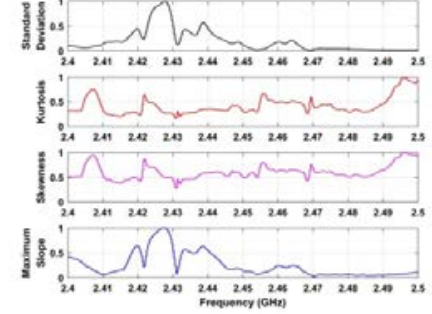

(e)

Fig. 22. Frequency estimates using the characteristics analysis algorithm at (a) $500 \mathrm{~m}$; (b) $1500 \mathrm{~m}$; (c) $2400 \mathrm{~m}$; (d) $2500 \mathrm{~m}$; and (e) $2800 \mathrm{~m}$ 


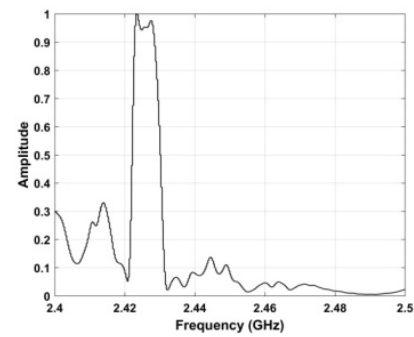

(a)

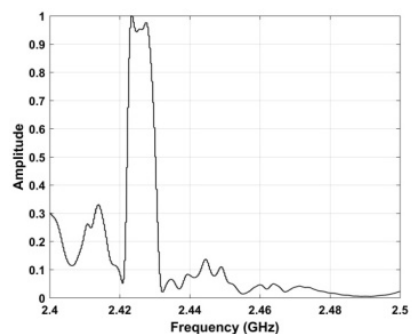

(b)

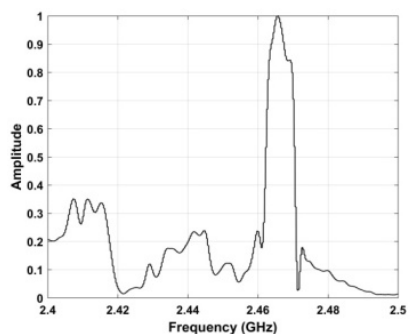

(c)

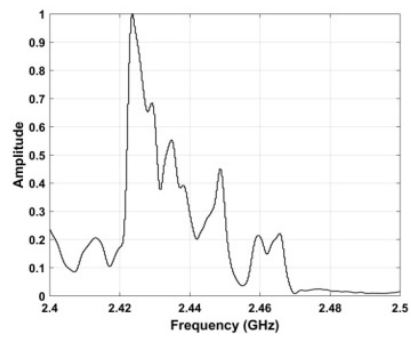

(d)

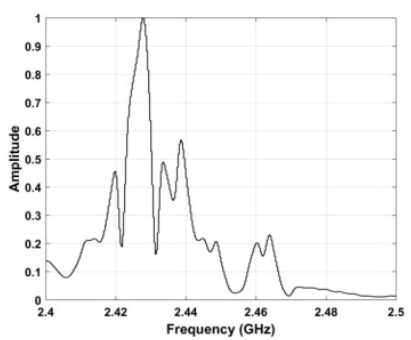

(e)

Fig. 23. Frequency estimates using the accumulation method at (a) $500 \mathrm{~m}$; (b) $1500 \mathrm{~m}$; (c) $2400 \mathrm{~m}$; (d) $2500 \mathrm{~m}$; and (e) $2800 \mathrm{~m}$

Using the results, the developed algorithm can provide accurate frequency estimates compared with the analyzed skewness and kurtosis. The frequency estimates of RF signals are within 2.42-2.43 GHz when the distances are $500 \mathrm{~m}, 1500 \mathrm{~m}, 2500 \mathrm{~m}$, and $2800 \mathrm{~m}$. Further, when the distance is $2400 \mathrm{~m}$, the frequency estimate is in the range of $2.46-2.47 \mathrm{GHz}$. All the estimated bandwidths of RF signals are about $10 \mathrm{MHz}$.

\subsection{Azimuth Estimation}

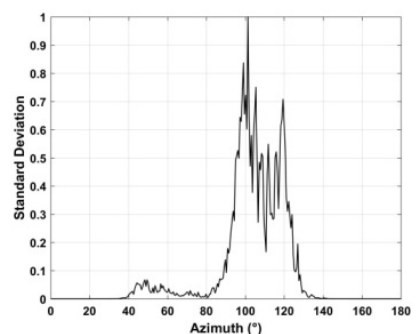

(a)

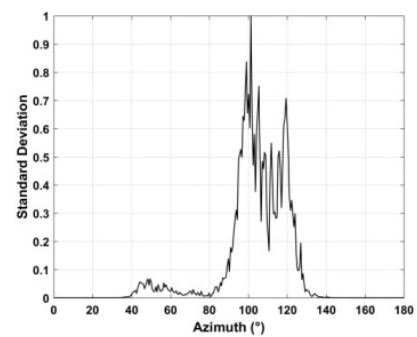

(b)

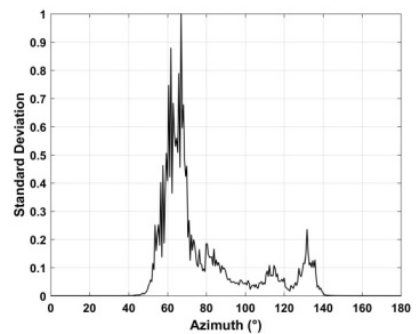

(c)

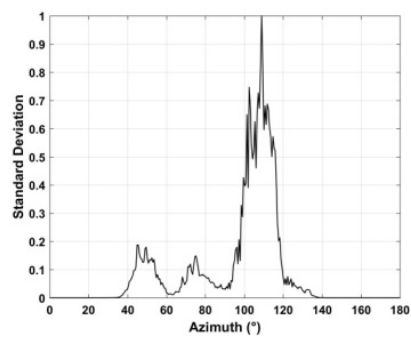

(d)

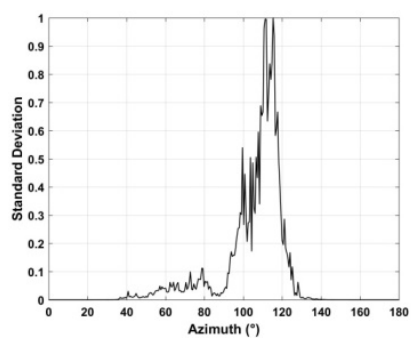

(e)

Fig. 24. Azimuth estimates using the developed method at (a) 500 m; (b) 1500 m; (c) 2400 m; (d) 2500 $\mathrm{m}$; and (e) $2800 \mathrm{~m}$ 
The azimuth estimates at different distances using the developed algorithm are shown in Fig. 24. From the results, the azimuth estimates are within $90^{\circ}-120^{\circ}$ when the distances are $500 \mathrm{~m}$, $1500 \mathrm{~m}, 2500 \mathrm{~m}$, and $2800 \mathrm{~m}$. When the range is $2400 \mathrm{~m}$, the azimuth estimate is within $50^{\circ}-80^{\circ}$. All results fit well with the acquired RF signals as shown in Figs. 18 and 20.

\subsection{Multiple Targets Detection}

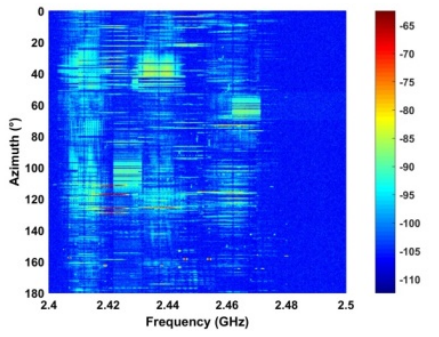

(a)

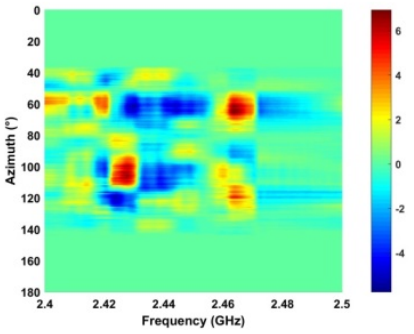

(b)

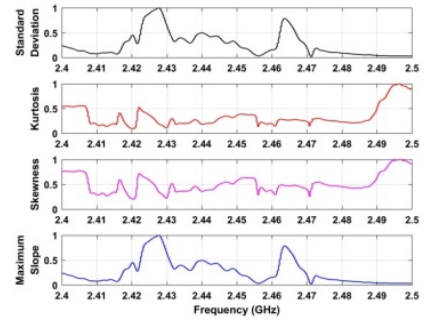

(c)

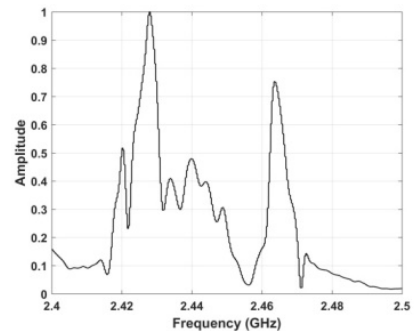

(d)

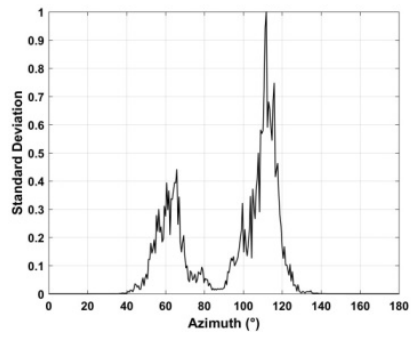

(e)

Fig. 25. The results from two targets (a) received RF signals; (b) results removing noises; (c) frequency estimates using the characteristics analysis method; (d) frequency estimates using the accumulation method; and (e) azimuth estimate using the developed algorithm.

The detection capability of multiple targets is validated using the radar system. Two drones are used as targets in the detection environments one is at a distance of $2400 \mathrm{~m}$, and another is at a distance of $2500 \mathrm{~m}$. The received RF signals are shown in Fig. 25(a), Fig. 25(b) shows the results after removing noises. Based on the results, two target areas are obvious to acquire, which provide the basis for the drone detection. The frequency estimates of the two drones are shown in Fig. 25(c) using the developed characteristics analysis algorithm. Fig. 25(d) provides the two frequency estimates acquired from the proposed accumulation method. Finally, the two azimuth estimates at different distances based on the developed algorithm are shown in Fig. 25(e). Based on the results, the azimuth estimate of one drone is within $90^{\circ}-120^{\circ}$, while another is within $50^{\circ}-80^{\circ}$.

\section{Conclusion}

This paper aims at the detection of aerial targets based on the passive radar. Using the passive radar, an improved algorithm is developed to identify drones. The noises affecting drone detection are suppressed effectively, and RF signals are improved based on the principal component analysis and automatic gain control algorithms. The EMD-based WT is used to reconstruct RF signals. The drones can be detected precisely. The azimuth information can be acquired based on the statistical theory. The monitoring system is validated and tested with different experiments conducted in stronger interference condition. 


\section{References}

[1] S. Claudia, B. Rohan, N. Francesco, G. Markus, Z. Jaap, "Review of the Current State of UAV Regulations,” Remote Sensing, vol. 9, no. 5, May 2017. Article (CrossRef Link)

[2] G. Zhou, V. Ambrosia, A. Gasiewski, "Foreword to the Special Issue on Unmanned Airborne Vehicle (UAV) Sensing Systems for Earth Observations," IEEE Transactions on Geoscience and Remote Sensing, vol. 47, no. 3, pp. 687-689, Mar. 2009. Article (CrossRef Link)

[3] F. Nex, F. Remondino, "UAV for 3D mapping applications: A review,” Applied Geomatics, vol. 6, no. 1, pp: 1-15, Mar. 2014. Article (CrossRef Link)

[4] G. Ding, Q. Wu, L. Zhang, Y. Lin, T.A. Tsiftsis, Y. Yao, “An amateur drone surveillance system based on cognitive internet of things,” IEEE Communications Magazine, vol. 56, no.1, pp. 29 - 35, Jan. 2018.

[5] Y. Zhao, J. Ma, X. Li, J. Zhang, "Saliency Detection and Deep Learning-Based Wildfire Detection in UAV Imagery,” Sensors, vol. 18, no. 3, p. 712. Feb. 2017. Article (CrossRef Link)

[6] J. Li, J. Chen, P. Wang, C. Li, "Sensor-Oriented Path Planning for Multiregion Surveillance with a Single Lightweight UAV SAR,” Sensors, vol. 18, no. 2, p. 548, Feb. 2018. Article (CrossRef Link)

[7] Sophie Crommelinck, Rohan Mark Bennett, Markus Gerke, Francesco Nex, "Review of automatic feature extraction from high-resolution optical sensor data for UAV-based cadastral mapping," Remote Sensing, vol. 8, no. 8, p. 689, Sep. 2016. Article (CrossRef Link)

[8] Remondino, F., "Heritage recording and 3D modeling with photogrammetry and 3D scanning," Remote Sensing, vol. 3, pp. 1104-1138, Mar. 2011.

[9] G. Bai, J. Liu, Y. Song, Y. Zuo, "Two-UAV Intersection Localization System Based on the Airborne Optoelectronic Platform,” Sensors, vol. 17, no. 1, p. 98, Jan. 2017. Article (CrossRef Link)

[10] S. Harwin, A. Lucieer, “Assessing the accuracy of georeferenced point clouds produced via multi-view stereopsis from unmanned aerial vehicle (UAV) imagery,” Remote Sensing, vol. 4, pp. 1573-1599, Apr. 2012. Article (CrossRef Link)

[11] I. Aicardi, F. Nex, M. Gerke, A.M. Lingua, "An image-based approach for the co-registration of multi-temporal UAV image datasets,” Remote Sensing, vol. 8, no. 9, p. 779, Sep. 2016. Article (CrossRef Link)

[12] F. Agüera-Vega, F. Carvajal-Ramírez, P. Martínez-Carricondo, “Assessment of photogrammetric mapping accuracy based on variation ground control points number using unmanned aerial vehicle,” Measurement, vol. 98, pp. 221-227. Jun. 2017.

[13] C. Yifeng, S. Kousuke, "Notice of Removal: Cooperative system for UAV and ground robot based on cognitive sharing," in Proc. of 2015 54th Annual Conference of the Society of Instrument and Control Engineers of Japan (SICE), pp. 28-30, Jul. 2015. Article (CrossRef Link)

[14] C. Li, X. Wang, "Jamming of unmanned aerial vehicle with GPS/INS integrated navigation system based on trajectory cheating," in Proc. of 2016 9th International Congress on Image and Signal Processing, BioMedical Engineering and Informatics (CISP-BMEI), pp. 15-17, Oct. 2016.

[15] K. Hartmann, K. Giles, "UAV exploitation: A new domain for cyber power," in Proc. of 2016 8th International Conference on Cyber Conflict (CyCon), Jun. 2016. Article (CrossRef Link)

[16] D.H. Shin, D.H. Jung, D.C. Kim, “A distributed FMCW radar system based on fiber-optic links for small drone detection,” IEEE Transactions on Instrumentation and Measurement, vol. 66, no. 2, Feb. 2017. Article (CrossRef Link)

[17] R. May, Y. Steinheim, P. Kvaløy, "Performance test and verification of an off-the-shelf automated avian radar tracking system,” Ecology and Evolution, vol. 7, no. 15, pp. 5930-5938, 2017. Article (CrossRef Link)

[18] J. Ren, X. Jiang, "Regularized 2-D complex-log spectral analysis and subspace reliability analysis of micro-Doppler signature for UAV detection,” Pattern Recognition, vol. 69, pp. 225-237, Sep. 2017.

[19] L. Xin, N. Xian, "Biological object recognition approach using space variant resolution and pigeon-inspired optimization for UAV,” Science China Technological Sciences, vol. 60, no. 10, pp. 1577-1584, Oct. 2017. 
[20] E.E. Case, A.M. Zelnio, B.O. Rigling, "Low-cost acoustic array for small UAV detection and tracking,” in Proc. of NAECON, Dayton, OH, USA, pp. 110-113, Jul. 2008. Article (CrossRef Link)

[21] K.S. Narendra, K. Parthasarathy, "Identification and control of dynamical systems using neural networks,” IEEE transactions on neural networks, vol. 1, no. 1, pp. 4-27, Jan. 1990. Article (CrossRef Link)

[22] L.X. Wang, "Design and Analysis of Fuzzy Identifiers of Nonlinear Dynamic Systems,” IEEE Transactions on Automatic Control, vol. 40, no. 1, pp. 11-23. Jan. 1995. Article (CrossRef Link)

[23] K.W. LLiff, “Parameter estimation for flight vehicles,” Journal of Guidance Control \& Dynamics. vol. 12, no. 5, pp. 609-622, May 1989. Article (CrossRef Link)

[24] R. Pintelon, J. Schoukens, “System Identification: A Frequency Domain Approach,” Wiley, IEEE Press, 2001. Article (CrossRef Link)

[25] R.W. Johnson, S. Jayaram, L. Sun, J. Zalewski, "Distributed processing kalman filter for automated vehicle parameter estimation-a case study," in Proc. of IASTED Intl Conf. on Applied Simulation and Modeling, pp. 270-276, 2010. Article (CrossRef Link)

[26] J. Suk, Y. Lee, S. Kim, H. Koo, J. Kim, "System identification and stability evaluation of an unmanned aerial vehicle from automated flight tests,” KSME International Journal, vol. 17, no. 5, pp. 654-667, May 2003. Article (CrossRef Link)

[27] S. Hengy, M. Laurenzis, S. Schertzer, A. Hommes, "Multimodal UAV detection: Study of various intrusion scenarios,” in Proc. SPIE, Warsaw, Poland, vol. 10434, p. 104340, Sep. 2017. Article (CrossRef Link)

[28] W.C. Liu, T.C. Wei, Y.S. Huang, "All digital synchronization for SC/OFDM mode of IEEE 802.15.3c and IEEE 802.11ad,” IEEE Transactions on Circuits and Systems I: Regular Papers, vol. 62, no. 2, pp. 545-553, Feb. 2015. Article (CrossRef Link)

[29] X. Liang, H. Zhang, T.A. Gulliver, "An Improved Algorithm for Through-wall Target Detection Using Ultra-wideband Impulse Radar,” IEEE Access, vol. 5, pp. 22101-22118, Dec. 2017. Article (CrossRef Link)

[30] X. Liang, H. Zhang, T.A. Gulliver, "Improved Denoising Method for Through-wall Vital Sign Detection Using UWB Impulse Radar,” Digital Signal Processing, vol. 74, pp. 72-93, Mar. 2018. Article (CrossRef Link)

[31] S. Dikmese, P.C. Sofotasios, M. Renfors, M. Valkama, "Subband Energy Based Reduced Complexity Spectrum Sensing Under Noise Uncertainty and Frequency-Selective Spectral Characteristics,” IEEE Transactions on Signal Processing, vol. 64, no. 1, pp. 131-145, Sep. 2015. Article (CrossRef Link)

[32] Y. Li, “A new single MCCCDTA based Wien-bridge oscillator with AGC,” AEU - International Journal of Electronics and Communications, vol. 66, no. 2, pp. 153-156, Feb. 2012. Article (CrossRef Link)

[33] X. Liang, H. Zhang, T.A. Gulliver, "Ultra-wide band impulse radar for life detection using wavelet packet decomposition,” Physical Communication, vol. 29, pp. 31-47, Aug. 2018. Article (CrossRef Link)

[34] X. Liang, H. Zhang, T.A. Gulliver, "Through-wall Human Being Detection Using UWB Impulse Radar,” EURASIP Journal on Wireless Communications and Networking, vol. 2018, no. 46, pp. 1-17, Feb. 2018. Article (CrossRef Link)

[35] D. Shahbaztabar, S. Alirezaee, M. Ahmadi, R. Heydari, “A MC-CDMA system based on orthogonal filter banks of wavelet transforms and partial combining," AEU - International Journal of Electronics and Communications, vol. 94, pp. 128-138, Sep. 2018. Article (CrossRef Link)

[36] S. Dikmese, P.C. Sofotasios, T. Ihalainen, M. Renfors, M. Valkama, "Efficient Energy Detection Methods for Spectrum Sensing Under Non-Flat Spectral Characteristics," IEEE Journal on Selected Areas in Communications, vol. 33, no. 5, pp.755-770, Oct. 2014. Article (CrossRef Link)

[37] S. Dikmese, Z. Ilyas, P.C. Sofotasios, M. Renfors, M. Valkama, "Sparse Frequency Domain Spectrum Sensing and Sharing Based on Cyclic Prefix Autocorrelation,” IEEE Journal on Selected Areas in Communications, vol. 35, no. 1, pp. 159-172, Jan. 2017. Article (CrossRef Link) 


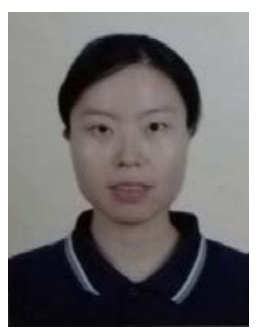

Xue Lv is an Associate Professor of Wuhan Huaxia University of Technology. She received the Master and Doctor degree from Wuhan University of Technology,Wuhan,China. Her current research interest includes wireless sensor network,automation and so on.

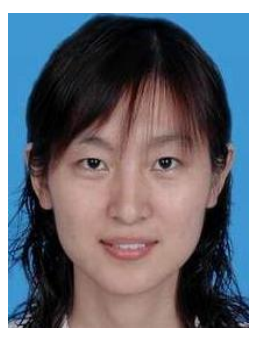

Zekun Wang is a lecturer of Wuhan Huaxia University of Technology. She received the Master degree from JINAN University. Her current research interests include automatic ide ntification technology, wireless communication and so on. 